\title{
Is sequential cranial ultrasound reliable for detection of white matter injury in very preterm infants?
}

\author{
Lara M. Leijser • Francisca T. de Bruïne • \\ Jeroen van der Grond • Sylke J. Steggerda • \\ Frans J. Walther • Gerda van Wezel-Meijler
}

Received: 28 August 2009/Accepted: 4 February 2010 /Published online: 6 March 2010

(C) The Author(s) 2010. This article is published with open access at Springerlink.com

\begin{abstract}
Introduction Cranial ultrasound (cUS) may not be reliable for detection of diffuse white matter (WM) injury. Our aim was to assess in very preterm infants the reliability of a classification system for WM injury on sequential cUS throughout the neonatal period, using magnetic resonance imaging (MRI) as reference standard.

Methods In 110 very preterm infants (gestational age $<32$ weeks), serial cUS during admission (median 8, range 4-22) and again around term equivalent age (TEA) and a single MRI around TEA were performed. cUS during admission were assessed for presence of WM changes, and contemporaneous cUS and MRI around TEA additionally for abnormality of lateral ventricles. Sequential cUS (from birth up to TEA) and MRI were classified as normal/ mildly abnormal, moderately abnormal, or severely abnormal, based on a combination of findings of the WM and lateral ventricles. Predictive values of the cUS classification were calculated.

Results Sequential cUS were classified as normal/mildly abnormal, moderately abnormal, and severely abnormal in, respectively, $22 \%, 65 \%$, and $13 \%$ of infants and MRI in, respectively, $30 \%, 52 \%$, and $18 \%$. The positive predictive value of the cUS classification for the MRI classification was high for severely abnormal WM (0.79) but lower for
\end{abstract}

\footnotetext{
L. M. Leijser - S. J. Steggerda · F. J. Walther ·

G. van Wezel-Meijler $(\square)$

Department of Pediatrics, Division of Neonatology, J6-S, Leiden University Medical Center,

P.O. Box 9600, 2300 RC Leiden, The Netherlands

e-mail: g.van_wezel-meijler@lumc.nl

F. T. de Bruïne $\cdot$ J. van der Grond

Department of Radiology, division of Neuroradiology,

Leiden University Medical Center,

P.O. Box 9600, 2300 RC Leiden, The Netherlands
}

normal/mildly abnormal $(0.67)$ and moderately abnormal (0.64) WM.

Conclusion Sequential cUS during the neonatal period detects severely abnormal WM in very preterm infants but is less reliable for mildly and moderately abnormal WM. MRI around TEA seems needed to reliably detect WM injury in very preterm infants.

Keywords Cranial ultrasound · MRI · Preterm infant . Brain - White matter injury

\section{Introduction}

Diffuse white matter injury is frequently encountered in very preterm infants [1-15]. It is reflected by signal changes in the white matter (WM) on magnetic resonance imaging (MRI) [2-14]. Concerns have been raised that cranial ultrasound (cUS) is not sensitive enough to detect diffuse WM injury, as the abnormalities may be subtle [2-10]. Preterm infants with diffuse WM injury are at risk for motor and cognitive impairment [11, 15]. Several authors have therefore suggested a standard MRI examination in all infants born very prematurely (gestational age (GA) $<32$ weeks) [6-9].

In preterm infants, WM injury has been associated with reduced WM and deep (i.e., basal ganglia and thalami) and cortical gray matter volumes and increased cerebrospinal fluid volumes around term equivalent age (TEA) [16-18]. However, previous cUS studies on WM injury in very preterm infants have only assessed changes within the WM but did not assess associated changes such as ventricular dilatation [3, 4, 6-8].

In this prospective study, we assess the reliability of a classification system for WM injury in very preterm infants, based on a combination of findings of the WM and lateral 
ventricles, on frequent, sequential high-quality cUS throughout the neonatal period, using an MRI classification system as reference standard.

\section{Materials and methods}

\section{Patients}

Very preterm infants (GA $<32$ weeks), admitted to the tertiary neonatal intensive care unit of the Leiden University Medical Center between May 2006 and October 2007, were eligible for a prospective neuroimaging study, assessing brain maturation and brain injury by cUS and MRI. The study was approved by the Medical Ethics Committee, and informed consent was obtained from the parents. Exclusion criteria were congenital anomalies of the central nervous system, severe other congenital anomalies, chromosomal and metabolic disorders, and neonatal meningitis.

Cranial ultrasound

\section{Image acquisition}

Serial cUS scans were performed by a team of experienced examiners, using an Aloka $\alpha 10$ scanner (Biomedic Nederland B.V., Almere, the Netherlands) with a special standardized preset and according to a standard protocol: scanning with a transducer frequency of $7.5 \mathrm{MHz}$ within $24 \mathrm{~h}$ of birth, at least weekly from the day of birth or admission until discharge or transfer to another hospital, and again on the day of the MRI examination around TEA [19, 20].

\section{Assessment}

All cUS examinations were assessed by two experienced investigators (LML with SJS (both $>5$ years of experience) or LML with GvWM (>15 years of experience)).

For this part of the study, special attention was paid to the WM. On the serial cUS scans performed during admission periventricular echodensities (PVE) were defined and classified according to van Wezel-Meijler et al., relating the echogenicity of the WM to that of the choroid plexus, and their appearance (homogenous or inhomogeneous) was noted (Fig. 1) [2, 21]. As, based on previous experience, PVE may still be seen at discharge and consequently the duration of PVE cannot be reliably assessed, we decided not to include the total duration of PVE in our WM classification. Subtle, symmetric echogenic areas in the frontal WM or adjacent and parallel to the atrium of the lateral ventricles were considered normal phenomena and not scored as PVE [21, 22].

Unilateral or asymmetric localized areas of high echogenicity within the WM were scored as focal WM echodensities. If coexisting with an intraventricular hemorrhage on the ipsilateral side, these mostly represent periventricular hemorrhagic infarction [23]. When in doubt whether an echodensity in the WM represented PVE or a focal echodensity, it was scored as inconclusive. Porencephalic cyst was defined as a large cystic lesion, communicating with the lateral ventricle [23].

On the cUS performed within several hours of the MRI (i.e., TEA-cUS), besides assessment of WM changes, the ventricular index (VI) was measured according to Levene, by an independent investigator who was not involved in patient care and unaware of the imaging findings [24]. A VI of $<13 \mathrm{~mm}$ was considered as normal/mildly abnormal, between 13 and $15 \mathrm{~mm}$ as moderately abnormal, and of $>15 \mathrm{~mm}$ as severely abnormal. In addition, on TEA-cUS, the shape of the lateral ventricles was visually assessed and graded as normal/mildly abnormal (i.e., normal or mildly abnormal shape), moderately abnormal (i.e., moderately abnormal shape, including irregular, plump or squareshaped ventricles; Fig. 2a), or severely abnormal (i.e., severely abnormal shape; Fig. 2b). In case of discordance, consensus was reached.

\section{WM classification}

The WM classification was scored by the same investigators (LML with SJS or LML with GvWM). In case of discordance, consensus was reached.

WM classification for serial cUS during admission:

- Normal/mildly abnormal: no PVE or homogeneous grade 1 PVE (Fig. 1a) [21]

- Moderately abnormal: inhomogeneous grade 1 PVE (regardless of duration), grade 2 PVE (regardless of appearance and duration), and/or small, localized cystic lesions (Fig. 1b) [21]

- Severely abnormal: multicystic lesions, focal WM echodensity, and/or porencephalic cyst [23]

WM classification for TEA-cUS:

- Normal/mildly abnormal: homogeneous WM and nor$\mathrm{mal} /$ mildly abnormal lateral ventricles, the VI being less than $13 \mathrm{~mm}$ with the shape normal or at the most mildly abnormal

- Moderately abnormal: inhomogeneous WM and/or small, localized cystic lesions and/or moderately abnormal lateral ventricles, the VI being 13-15 $\mathrm{mm}$ and/or the shape moderately abnormal (Fig. 2a)

- Severely abnormal: multicystic lesions and/or focal WM echodensity and/or porencephalic cyst and/or severely abnormal lateral ventricles, the VI being over $15 \mathrm{~mm}$ and/or the shape severely abnormal (Fig. 2b).

The WM score of the MRI-cUS was based on the most severe changes. 
Fig. 1 a Coronal (left) and parasagittal (right) cUS of preterm infant (GA 27.9 weeks), scanned at PMA 31.1 weeks, showing normal echogenicity of periventricular WM (WM score of serial cUS during admission: normal/mildly abnormal)

b Coronal (left) and parasagittal (right) cUS of preterm infant (GA 28.0 weeks), scanned at PMA 32.3 weeks, showing homogeneous PVE in parietooccipital WM on the left and inhomogeneous PVE in parietooccipital WM on the right (arrows); (WM score of serial cUS during admission: normal/ mildly abnormal)
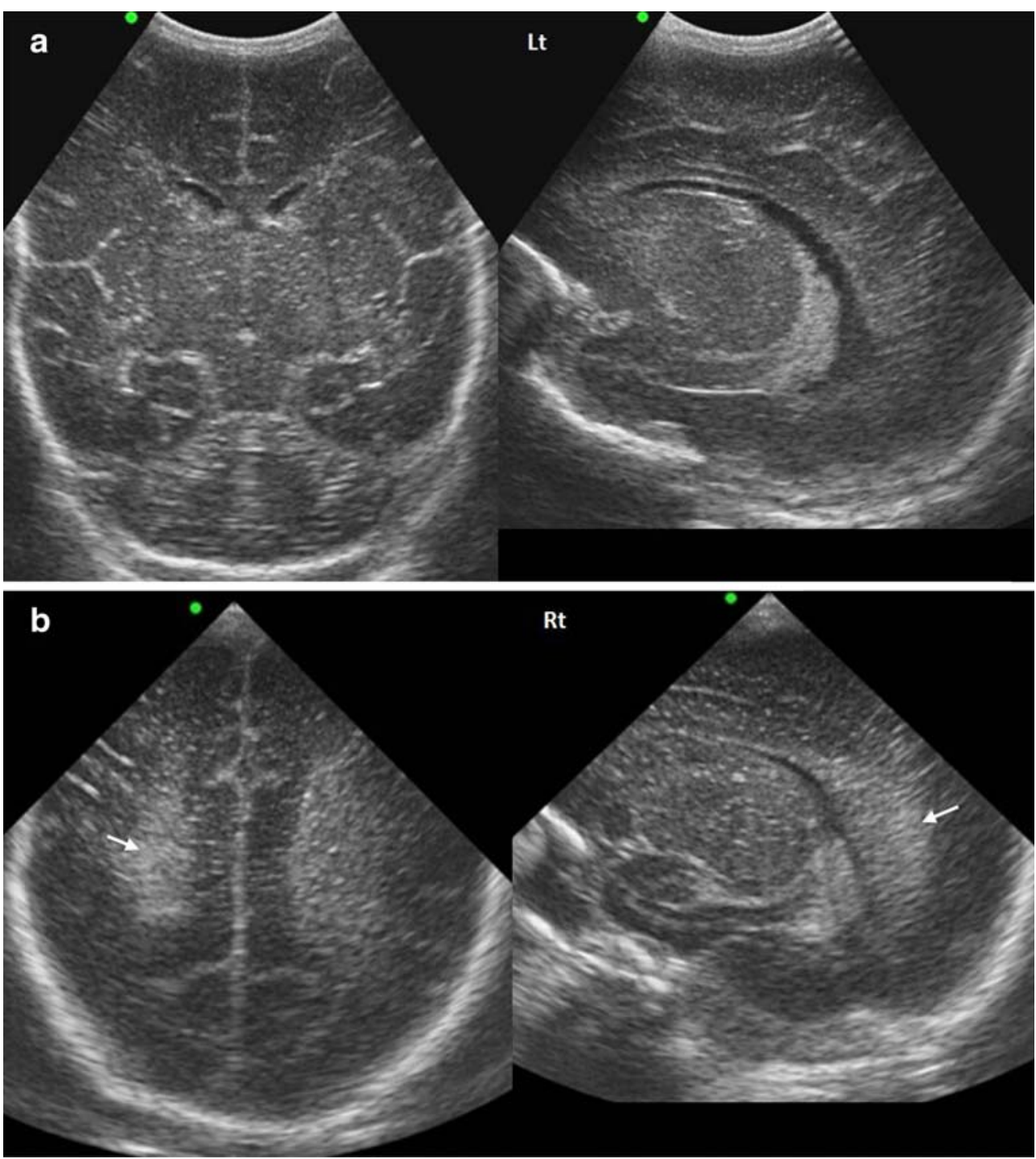

Fig. 2 a Coronal cUS of preterm infant (GA 28.0 weeks), scanned at PMA 42.0 weeks, showing moderately dilated lateral ventricles (TEA-cUS WM score: moderately abnormal). b. Coronal cUS of preterm infant (GA 30.3 weeks), scanned at PMA 41.9 weeks, showing severe lateral ventricular dilatation (TEA-cUS WM score: severely abnormal). Poor image quality is due to small size of fontanel
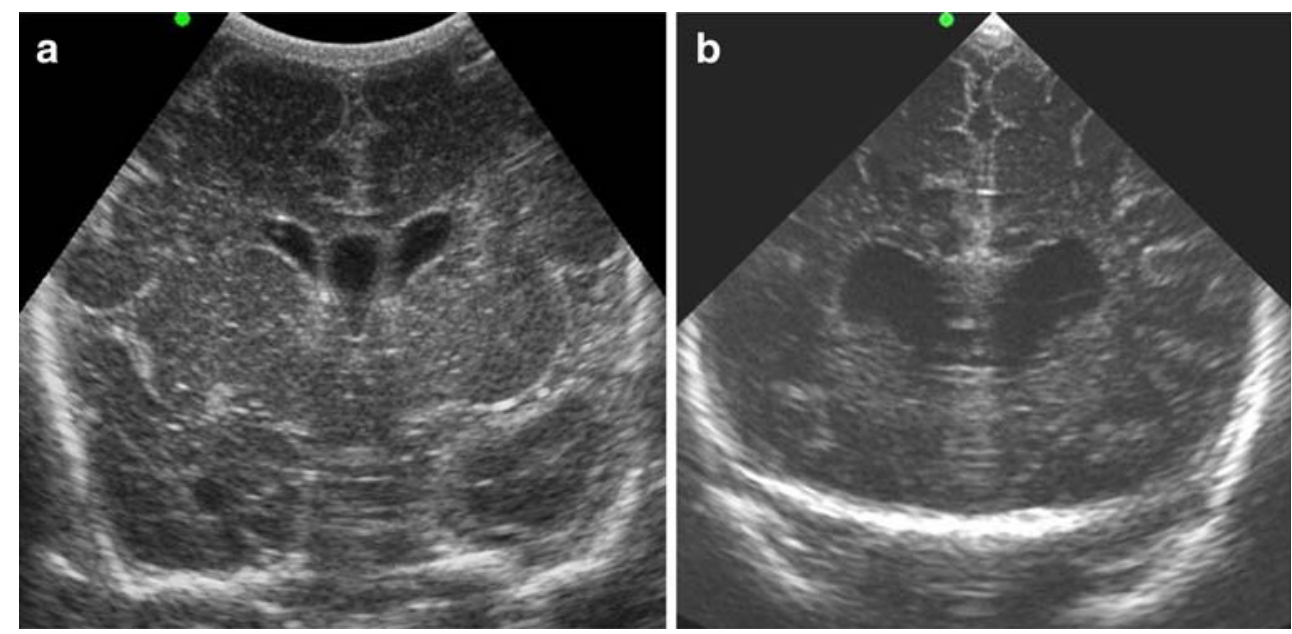
The WM classification for the serial cUS during admission combined with the TEA-cUS (i.e., sequential cUS) was based on the most severe changes over time.

\section{MRI}

\section{Image acquisition}

MRI examinations were performed in all very preterm infants according to a standard protocol for imaging the newborn infant's brain, using a 3.0-T Philips MR system (Philips Medical Systems, Best, the Netherlands) as recently described [25]. In summary, scans included at least $\mathrm{T}_{1}$-weighted three-dimensional images (repetition time/echo time (TR/TE) 9.7/4.6 ms), allowing reconstruction in every desired orientation, $\mathrm{T}_{2}$-weighted images (TR/ TE 6,269/120 ms), diffusion-weighted images (TR/TE 2,406/64 ms), and susceptibility weighted images (TR/TE $735 / 16 \mathrm{~ms}$ ) in the transverse plane. The MRI examinations were performed around TEA, preferably between 40- and 44-week postmenstrual age (PMA; i.e., TEA-MRI). For infants who were still unstable and/or ventilator dependent around that age, MRI was postponed. For this part of the study, the $T_{1}$ - and $T_{2}$-weighted sequences were analyzed.

\section{Visual assessment}

All MRI examinations were assessed by two experienced investigators (LML with FTdB or SJS with FTdB, the latter being a pediatric neuroradiologist with $>15$ years of experience), who were blinded to the cUS findings.

Special attention was paid to the brain WM. Punctate white matter lesions (PWML) were defined as small areas of high signal on $\mathrm{T}_{1}$-weighted images, with mostly low signal on $T_{2}$-weighted images (Fig. 3) [3, 20]. Diffuse and excessive high signal intensity (DEHSI) was defined as

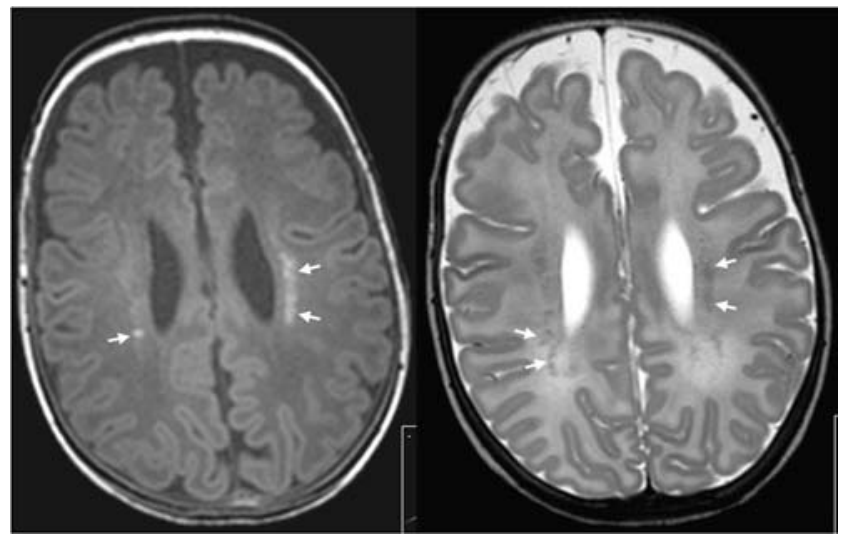

Fig. 3 Transverse $\mathrm{T}_{1^{-}}$(left) and $\mathrm{T}_{2}$-weighted (right) MRI at highventricular level of preterm infant (GA 26.9 weeks), scanned at PMA 42.7 weeks, showing bilateral, multiple PWML (arrows) in a linear distribution parallel to the LV. Also showing dilated, irregularly shaped lateral ventricles and widening of extracerebral spaces (TEAMRI WM score: moderately abnormal)
Fig. 4 Transverse $T_{2}$-weighted MRI at midventricular level of preterm infant (GA 30.6 weeks), scanned at PMA 42.3 weeks, showing homogeneous SI increase (DEHSI) in periventricular and subcortical WM (TEA-MRI WM score: normal/mildly abnormal)

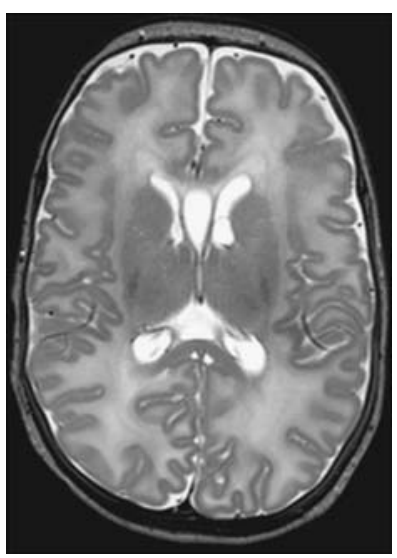

areas of excessive high signal intensity (SI) diffusely distributed within the periventricular and/or subcortical $\mathrm{WM}$ on $\mathrm{T}_{2}$-weighted images (Fig. 4) $[3-5,11,13,20]$.

From the $\mathrm{T}_{1}$-weighted three-dimensional images, coronal reconstructions were obtained and the VI was measured in the coronal plane showing the third ventricle. The total width of the lateral ventricles was measured and divided by 2 , in analogy to measurements of the VI on cUS. The shape of the lateral ventricles was assessed visually and graded as normal/mildly abnormal (Fig. 5a), moderately abnormal (Fig. 5b), or severely abnormal (Fig. 5c) as described for TEA-cUS. In case of discordance, consensus was reached.

\section{WM classification}

The WM classification was adapted from Sie et al. and scored by the same investigators. This was based on the most severe changes [2]. In case of discordance, consensus was reached.

- Normal/mildly abnormal: normal-appearing WM, homogeneous DEHSI or few $(\leq 6)$ PWML and normal/ mildly abnormal lateral ventricles, the VI being less than $13 \mathrm{~mm}$ with the shape normal or at the most mildly abnormal (Figs. 4 and 5a)

- Moderately abnormal: multiple (>6) PWML and/or small localized cystic lesions and/or inhomogeneous DEHSI and/or moderately abnormal lateral ventricles, the VI being 13-15 $\mathrm{mm}$ and/or the shape moderately abnormal (Figs. 3 and $5 b$ )

- Severely abnormal: extensive or diffuse inhomogeneous SI changes and/or hemorrhagic or cystic lesions involving the periventricular and/or subcortical WM and/or severely abnormal lateral ventricles, the VI being over $15 \mathrm{~mm}$ and/or the shape severely abnormal (Fig. 5c)

\section{Data analysis}

Statistical analyses were performed using SPSS (version 14.0; SPSS Inc., Chicago, IL, USA). Predictive values of 

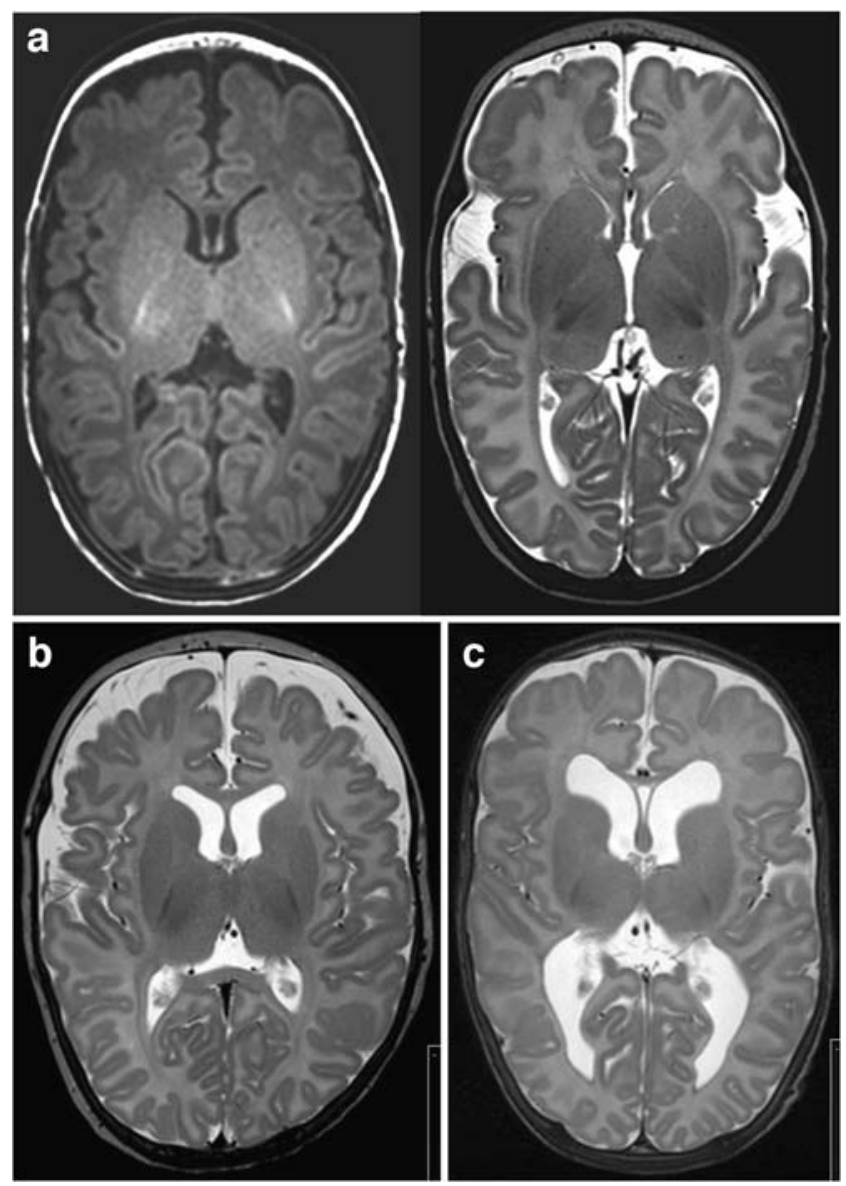

Fig. 5 a Transverse $\mathrm{T}_{1^{-}}$(left) and $\mathrm{T}_{2}$-weighted (right) $\mathrm{MRI}$ at midventricular level of preterm infant (GA 27.1 weeks), scanned at PMA 42.1 weeks, showing normal signal in WM. Also showing normal size and shape of lateral ventricles (TEA-MRI WM score: normal/mildly abnormal). b Transverse $\mathrm{T}_{2}$-weighted MRI at midventricular level of preterm infant (GA 27.9 weeks), scanned at PMA 43.1 weeks, showing moderately dilated and abnormally squareshaped lateral ventricles (TEA-MRI WM score: moderately abnormal). c Transverse $T_{2}$-weighted MRI at midventricular level of preterm infant (GA 31.6 weeks), scanned at PMA 43.4 weeks, showing severely dilated and abnormally shaped lateral ventricles due to volume loss of the WM (TEA-MRI WM score: severely abnormal) the WM classification of TEA-cUS and of sequential cUS for the WM classification of TEA-MRI were calculated. As the signal of the WM and of WM lesions on MRI changes with increasing PMA and, therefore, may be different around TEA (PMA 40-44 weeks) than beyond TEA (PMA $>44$ weeks), we first calculated the predictive values for the total group of very preterm infants and subsequently only for the group of infants with TEA-cUS and TEA-MRI between 40- and 44-week PMA.

\section{Results}

Patients

During the study period, 182 very preterm infants were eligible for the study, of which 130 infants (80 male) were included (Fig. 6). Fifty-two infants were excluded from the study; three because of structural brain abnormalities and 49 because informed parental consent was not obtained. Reasons for not obtaining consent included transfer to another hospital or death within a very short period of birth, rejection of participation, and practical problems such as language barrier and travel distance to the hospital. Median GA and birth weight of included infants were 29.0 (range 25.6-31.9) weeks and 1,141 (520-1,960) g. There were no significant differences in GA and birth weight between infants with and without informed consent.

In all 130 infants, serial cUS scans were performed during admission (median 8, range 4-22). In 20 infants, no or inadequate TEA-cUS and TEA-MRI were obtained. In the remaining 110 infants (68 male), contemporaneous cUS and MRI were obtained at a median PMA of 43.4 (40.155.9) weeks. In 69 infants, this was around TEA (PMA 40-44 weeks) and in 41 infants between 44.0- and 55.9week PMA.
Fig. 6 Flow diagram showing the number of infants eligible for the study, the number of infants included and not included in the study, and the final number of infants with sequential cUS and MRI between 40and 44-week PMA ( $n$, number of infants)

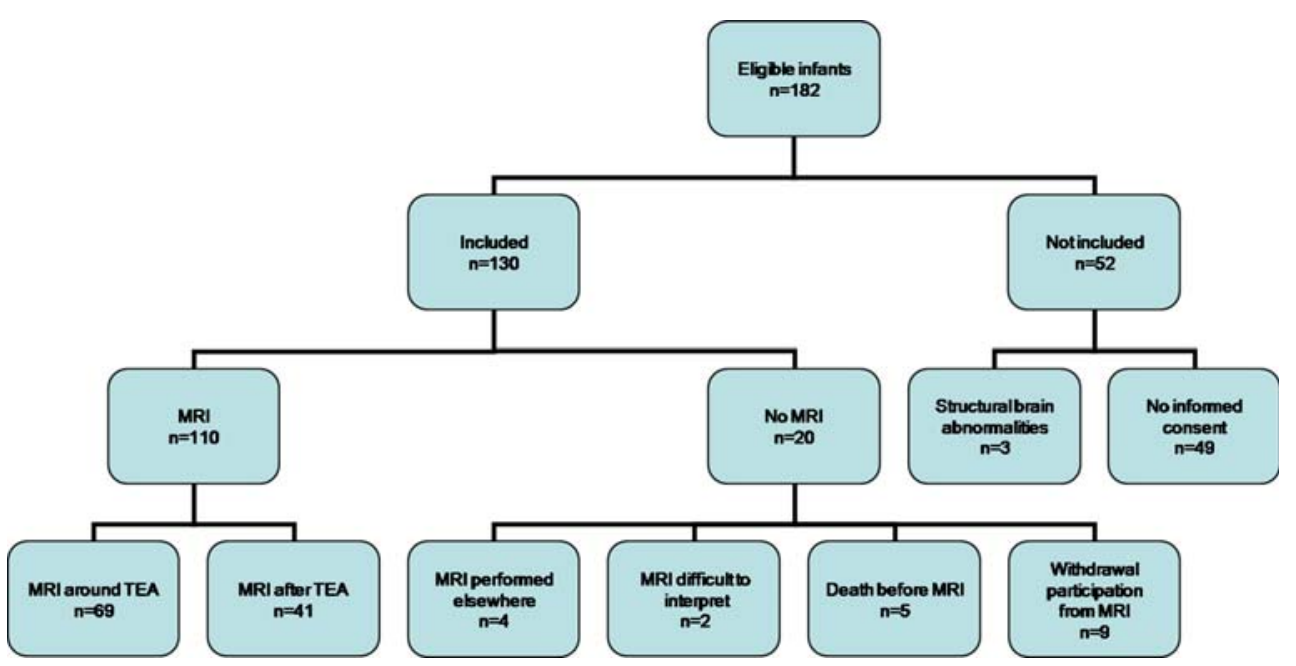




\section{Cranial ultrasound}

The distribution of the WM classifications for serial cUS during admission (based on WM changes), TEA-cUS (based on WM changes and size and shape of lateral ventricles), and sequential cUS (based on the most severe classification for serial cUS during admission and TEAcUS) is shown in Table 1 . Table 2 shows the distribution of WM findings that determined the WM classifications.

\section{MRI}

The distribution of the WM classification for TEA-MRI (based on WM changes and size and shape of lateral ventricles) is shown in Table 1. Table 2 shows the distribution of WM findings that determined the WM classification.

\section{Relation between cUS and MRI}

Predictive values of the WM classifications of TEA-cUS and of sequential cUS for the WM classification of TEAMRI for the total group of preterm infants $(n=110)$ are presented in Table 3. This table shows high positive predictive values of cUS for MRI in infants with severely abnormal WM but lower positive predictive values in infants with normal/mildly abnormal and moderately abnormal WM on cUS.

Predictive values of the WM classifications of TEA-cUS and of sequential cUS for the WM classification of TEAMRI for the subgroup of infants with TEA-cUS and TEAMRI between 40- and 44-week PMA $(n=69)$ are presented in Table 4. These predictive values do not differ from those for the total group of infants.

\section{Discussion}

To our knowledge, this is the first prospective study comparing WM injury on cUS and MRI (reference standard) in very preterm infants based on newly designed classification systems, not only including changes within the WM but also scoring other changes in the brain thought to be related to WM injury.
The most important finding of this study is that the positive predictive value of both the TEA-cUS and the sequential cUS WM classification for the TEA-MRI WM classification was high for severely abnormal WM; nearly all infants classified as severely abnormal for cUS were also classified as severely abnormal for MRI. However, cUS was less predictive for the moderately abnormal and normal/mildly abnormal groups.

There are several possible explanations for these results. MRI was performed with a 3.0-T MR system, providing a high resolution that cannot be obtained with ultrasonography. Using this high field strength and a special neonatal imaging protocol, small lesions, including very small punctate WM lesions (PWML), are depicted [20]. In addition, in accordance with Sie et al., we used 6 as the cutoff number for "few" (mildly abnormal WM) or "multiple" (moderately abnormal WM) PWML on MRI, which is rather arbitrary [2]. In 10/12 infants whose MRI classification was based on "multiple" PWML, just over six lesions were detected, mostly isolated in organization and located in the periventricular WM at the level of the centrum semiovale and/or adjacent to the optic radiation. Only in two infants, the number of PWML was considerably higher and lesions were more widely distributed in the WM and organized linearly and/or in clusters. In this respect, we may have overrated the severity of WM changes on MRI in several infants. Finally, when only the anterior fontanel is used as acoustic window, the occipital region is not easy to visualize with cUS. For this study, we did not additionally use the posterior fontanel, while this could have improved visualization of WM changes in the occipital region [19].

cUS not only underestimated WM injury (Fig. 7) but seemed to overestimate WM injury in some cases. This can be explained by the transient nature of some WM changes and the fact that the WM classification of sequential cUS was based on the most severe changes over time [20].

Combining the WM classification of serial cUS during admission with that of TEA-cUS (i.e., sequential cUS) improved the predictive value of cUS in case of normal/ mildly abnormal WM but decreased it for moderately abnormal WM.

The results of this study may arouse the erroneous suggestion that frequent, serial cUS performed during
Table 1 Distribution of the WM classifications for serial cUS during admission (adm-cUS), TEA-cUS, sequential cUS, and TEAMRI ( $n$, number of infants).

\begin{tabular}{lcccr}
\hline WM classification & \multicolumn{4}{l}{ Imaging technique, $n$ (\% of total number) } \\
\cline { 2 - 5 } & Adm-cUS & TEA-cUS & Sequential cUS & TEA-MRI \\
\hline Normal/mild & $27(24.5)$ & $56(51)$ & $24(22)$ & $33(30)$ \\
Moderate & $75(68.2)$ & $40(36)$ & $72(65)$ & $57(52)$ \\
Severe & $8(7.3)$ & $14(13)$ & $14(13)$ & $20(18)$ \\
\hline
\end{tabular}


Table 2 Distribution of WM changes and abnormalities of lateral ventricles determining the WM classification of serial cUS during admission (adm-cUS), TEA-cUS, and TEA-MRI.

\begin{tabular}{|c|c|c|c|}
\hline WM classification & & Findings determining WM classification & Number of infants \\
\hline \multirow[t]{7}{*}{ Adm-cUS } & \multirow[t]{2}{*}{ Normal/mild } & No PVE & 17 \\
\hline & & Homogeneous grade $1 \mathrm{PVE}$ & 10 \\
\hline & \multirow[t]{3}{*}{ Moderate } & Inhomogeneous grade $1 \mathrm{PVE}$ & 57 \\
\hline & & Grade 2 PVE & 12 \\
\hline & & Small, localized cystic lesions & 6 \\
\hline & \multirow[t]{2}{*}{ Severe } & Multicystic lesions & 1 \\
\hline & & PVHI & 7 \\
\hline \multirow[t]{5}{*}{ TEA-cUS } & Normal/mild & Homogeneous WM and normal/mildly abnormal LV & 56 \\
\hline & \multirow[t]{3}{*}{ Moderate } & Inhomogeneous WM & 3 \\
\hline & & Moderately abnormal LV & 31 \\
\hline & & Inhomogeneous WM and moderately abnormal LV & 6 \\
\hline & Severe & Severely abnormal LV & 14 \\
\hline \multirow[t]{6}{*}{ TEA-MRI } & \multirow[t]{2}{*}{ Normal/mild } & Normal WM/homogeneous DEHSI and normal/mildly abnormal LV & 30 \\
\hline & & Few PWML and normal/mildly abnormal LV & 3 \\
\hline & \multirow[t]{3}{*}{ Moderate } & Multiple PWML & 4 \\
\hline & & Moderately abnormal LV & 45 \\
\hline & & Multiple PWML and moderately abnormal LV & 8 \\
\hline & Severe & Severely abnormal LV & 20 \\
\hline
\end{tabular}

DEHSI diffuse and excessive high signal intensity, $L V$ lateral ventricles, $P V E$ periventricular echodensities, $P V H I$ periventricular hemorrhagic infarction, $P W M L$ punctate white matter lesions

admission insufficiently contributes to detection of WM injury in very preterm infants. However, our classification systems are used to grade the severity of WM injury and do not provide information on separate imaging findings or the evolution of lesions. By substantially limiting the number of cUS examinations during admission, details on (transient) WM changes will be lost, the evolution of lesions cannot be followed, and distinction between several forms of WM injury (i.e., focal or more diffuse) may not be possible, as only the end stages of WM injury are visualized and, for instance, focal cysts may have resolved by TEA. By restricting cUS to the early neonatal period and TEA, as has been suggested, no information will be available on the evolution of lesions, lesions may remain undetected, and their severity may be underestimated [26].
Our results are in agreement with those of others comparing WM findings on cUS and MRI, showing that MRI is more sensitive for detecting (particularly subtle) WM changes than cUS [2-10, 26, 27]. In addition, in most cases with WM injury that was detected by cUS, MRI demonstrated the exact site and extent of lesions more precisely, which is in agreement with previous findings [14].

We appreciate some limitations of our study. Firstly, the most stable infants with (nearly) normal cUS findings were discharged sooner than those being less stable and/or having more severe findings. We may therefore have missed progression towards more severe changes on cUS in some infants with minor changes or changes developing after discharge. This may have biased our results and
Table 3 Predictive values of the WM classifications of TEA-cUS and of sequential cUS for the WM classification of TEA-MRI for the total group of preterm infants.

$N P V$ negative predictive value, $P P V$ positive predictive value

\begin{tabular}{llcccc}
\hline \multirow{2}{*}{ cUS WM classification } & \multicolumn{4}{l}{ Predictive values for TEA-MRI WM classification } \\
\cline { 3 - 5 } & & Sensitivity & Specificity & PPV & NPV \\
\hline \multirow{2}{*}{ TEA-cUS } & Normal/mild & 0.94 & 0.68 & 0.55 & 0.96 \\
& Moderate & 0.51 & 0.79 & 0.73 & 0.60 \\
& Severe & 0.55 & 0.97 & 0.79 & 0.91 \\
\multirow{3}{*}{ Sequential cUS } & Normal/mild & 0.48 & 0.90 & 0.67 & 0.80 \\
& Moderate & 0.81 & 0.51 & 0.64 & 0.71 \\
& Severe & 0.55 & 0.97 & 0.79 & 0.93 \\
\hline
\end{tabular}


Table 4 Predictive values of the WM classifications of TEA-cUS and sequential cUS for the WM classification of TEA-MRI for the subgroup of infants with TEA-cUS and TEA-MRI between 40- and 44-week PMA.

$N P V$ negative predictive value, $P P V$ positive predictive value

\begin{tabular}{llcccc}
\hline \multirow{2}{*}{ cUS WM classification } & \multicolumn{3}{l}{ Predictive values for TEA-MRI WM classification } \\
\cline { 3 - 5 } & & Sensitivity & Specificity & PPV & NPV \\
\hline \multirow{2}{*}{ TEA-cUS } & Normal/mild & 0.95 & 0.60 & 0.50 & 0.97 \\
& Moderate & 0.46 & 0.79 & 0.71 & 0.55 \\
& Severe & 0.40 & 0.98 & 0.80 & 0.91 \\
\multirow{3}{*}{ Sequential cUS } & Normal/mild & 0.44 & 0.90 & 0.67 & 0.76 \\
& Moderate & 0.83 & 0.42 & 0.62 & 0.68 \\
& Severe & 0.40 & 0.98 & 0.80 & 0.91
\end{tabular}

negatively influenced the reliability of cUS as cUS was not very reliable in infants with normal/mildly abnormal WM. Secondly, as in half (44/89) of the infants with PVE on cUS these were seen on the last cUS performed before discharge but no longer on TEA-cUS, we were not able to reliably assess the total duration of PVE. The total duration of PVE was therefore not included in the WM classification. We only performed a single MRI examination around TEA. Therefore, while cUS also reflected early and/or transient WM changes and the evolution of changes, MRI reflected only the later stages of WM injury. In more than one third of the infants, MRI was performed after TEA, at a time when the signal of the WM may have changed. However, the predictive values of the WM classification of cUS for that of MRI calculated for the subgroup of preterm infants with TEA-cUS and TEA-MRI between 40- and 44-week PMA did not differ from those for the total group of infants. Besides, due to ongoing brain maturation, the signal of the WM increases on $\mathrm{T}_{1}$ - and decreases on $\mathrm{T}_{2}$-weighted images, while it generally decreases on $T_{1}$ - and increases on $\mathrm{T}_{2}$-weighted images due to diffuse injury. We therefore feel that including infants scanned beyond TEA has not influenced our data. We only measured the width of the frontal horns of the lateral ventricles, while in some cases widening of the lateral ventricles may be more prominent posteriorly. However, by also visually assessing the shape of the lateral ventricles, we probably did not overlook other changes to the lateral ventricles. As reference values for VI in preterm infants around TEA are unavailable, the grouping of VI in the WM classification was based on VI values obtained by us in low-risk (near) term neonates (unpublished data). However, as the same grouping was used for TEA-cUS and TEA-MRI, we feel that this will probably not have influenced our results. We chose not to exclude infants with intraventricular hemorrhage, as these may well have concomitant WM injury. Nine infants with intraventricular hemorrhage complicated by post hemorrhagic ventricular dilatation were therefore included. Due to the ventricular dilatation, these nine infants may have been allocated to a more severe white matter classification group. As the VI was measured both on TEA-cUS and TEA-MRI, this will probably not have influenced our results. In addition, in our WM classification, focal echodensities, mostly diagnosed as PVHI, and porencephalic cysts were classified as severely abnormal, regardless of their size, while bilateral, inhomogeneous PVE not evolving into multicystic lesions were classified as moderately abnormal. One may question this classification, as widespread
Fig. 7 Coronal (left) and parasagittal (right) cUS of preterm infant (GA 26.9 weeks), scanned at PMA 29.1 weeks, showing only mild, homogeneous PVE bilaterally in the parietal WM; the cUS of this infant performed around TEA (PMA 42.7 weeks) showed normal WM and mildly abnormal size and shape of lateral ventricles (sequential cUS WM score: normal/mildly abnormal). However, the MRI of the same infant (see Fig. 3) showed bilateral, multiple PWML and dilated, irregularly shaped lateral ventricles (TEA-MRI WM score: moderately abnormal)

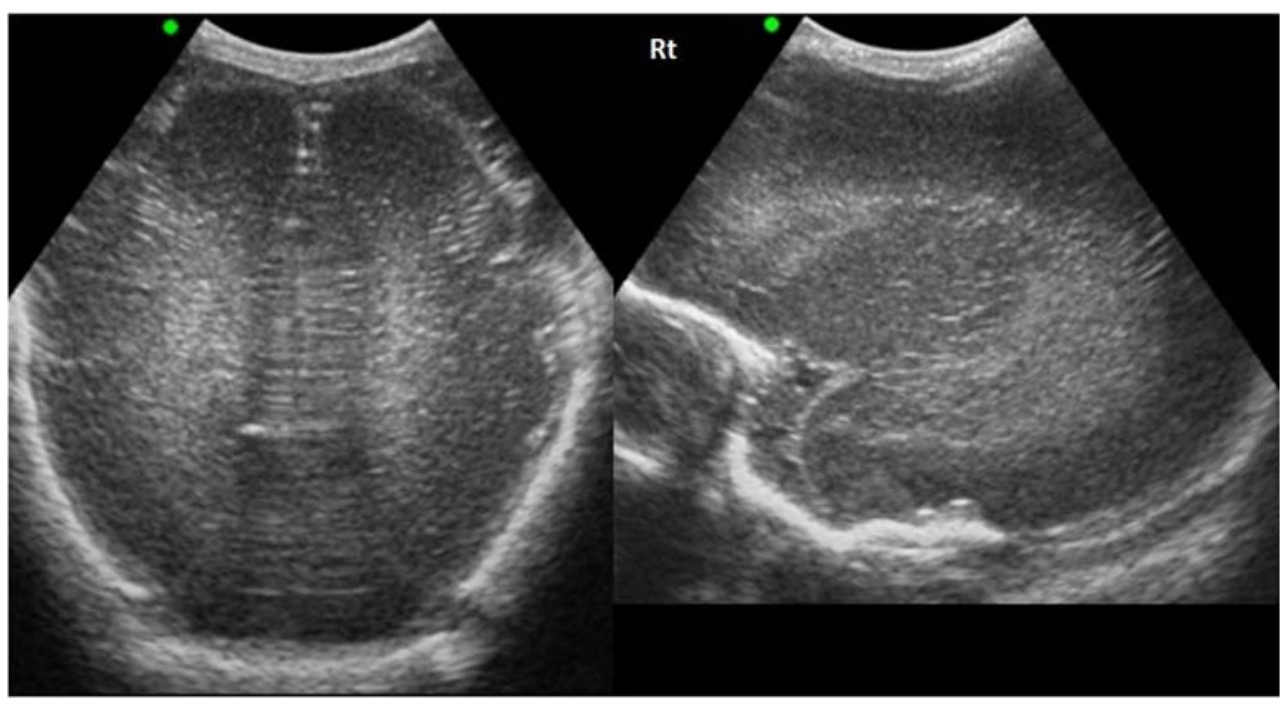


inhomogeneous PVE may be more serious than a small focal echodensity. However, also for the MRI classification, porencephalic cysts were classified as severely abnormal. We therefore feel this will not significantly have influenced our results. Brain imaging findings (cUS and MRI) were scored by two experienced investigators. This was done in consensus, and no interobserver agreement was calculated. Finally, we are not yet informed on the clinical significance of our WM classifications and the implications of the milder and more subtle WM changes, including PVE without cystic evolution on cUS and PWML and more diffuse SI changes on MRI, for neurological outcome. Follow-up data are currently obtained.

In conclusion, sequential cUS, even when performed frequently and being of good quality, may underestimate WM injury in very preterm infants. Therefore, while awaiting clinical follow-up data on our and other study populations, routine MRI around TEA in addition to serial cUS during admission seems needed in infants born very prematurely.

Future studies on WM injury in preterm infants should focus on the clinical significance of the WM classifications and of the separate changes, both on cUS and MRI, indicative of WM injury.

Acknowledgements We are grateful to neonatologists and staff of the pediatric ambulatory unit and MRI department of the Leiden University Medical Center and to B. van Kooij, University Medical Center Utrecht, for their helpful contributions to this project. We thank W. Teeuwisse for technical support and M. de Waard for the VI measurements.

Lara M. Leijser was supported by a grant from ZonMw, the Netherlands Organization for Health Research and Development (grant number 920-03-388).

Conflict of interest statement We declare that we have no conflict of interest.

Open Access This article is distributed under the terms of the Creative Commons Attribution Noncommercial License which permits any noncommercial use, distribution, and reproduction in any medium, provided the original author(s) and source are credited.

\section{References}

1. Paneth N, Rudelli R, Monte W, Rodriguez E, Pinto J, Kairam R, Kazam E (1990) White matter necrosis in very low birth weight infants: neuropathologic and ultrasonographic findings in infants surviving six days or longer. J Pediatr 16:975-984

2. Sie LTL, van der Knaap MS, van Wezel-Meijler G, Taets van Amerongen AH, Lafeber HN, Valk J (2000) Early MR features of hypoxic-ischemic brain injury in neonates with periventricular densities on sonograms. AJNR Am J Neuroradiol 21:852-861

3. Maalouf EF, Duggan PJ, Counsell SJ, Rutherford MA, Cowan F, Azzopardi D, Edwards AD (2001) Comparison of findings on cranial ultrasound and magnetic resonance imaging in preterm infants. Pediatrics 107:719-727
4. Childs AM, Cornette L, Ramenghi LA, Tanner SF, Arthur RJ, Martinez D, Levene MI (2001) Magnetic resonance and cranial ultrasound characteristics of periventricular white matter abnormalities in newborn infants. Clin Radiol 56:647-655

5. Cornette LG, Tanner SF, Ramenghi LA, Miall LS, Childs AM, Arthur RJ, Martinez D, Levene MI (2002) Magnetic resonance imaging of the infant brain: anatomical characteristics and clinical significance of punctate lesions. Arch Dis Child Fetal Neonatal Ed 86:F171-F177

6. Miller SP, Cozzio CC, Goldstein RB, Ferriero DM, Partridge JC, Vigneron DB, Barkovich AJ (2003) Comparing the diagnosis of white matter injury in premature newborns with serial MR imaging and transfontanel ultrasonography findings. AJNR Am J Neuroradiol 24:1661-1669

7. Inder TE, Anderson NJ, Spencer C, Wells S, Volpe JJ (2003) White matter injury in the premature infant: a comparison between serial cranial sonographic and MR findings at term. AJNR Am J Neuroradiol 24:805-809

8. Debillon T, N'Guyen S, Muet A, Quere MP, Moussaly F, Roze JC (2003) Limitations of ultrasonography for diagnosing white matter damage in preterm infants. Arch Dis Child Fetal Neonatal Ed 88: F275-F279

9. Mirmiran M, Barnes PD, Constantinou KK, JC FBE, Hintz SR, Ariagno RL (2004) Neonatal brain magnetic resonance imaging before discharge is better than serial cranial ultrasound in predicting cerebral palsy in very low birth weight preterm infants. Pediatrics 114:992-998

10. Rademaker KJ, Uiterwaal CSPM, Beek FJA, van Haastert IC, Lieftink AF, Groenendaal F, Grobbee DE, de Vries LS (2005) Neonatal cranial ultrasound versus MRI and neurodevelopmental outcome at school age in children born preterm. Arch Dis Child Fetal Neonatal Ed 90:F489-F493

11. Dyet LE, Kennea N, Counsell SJ, Maalouf EF, Ajayi-Obe M, Duggan PJ, Harrison M, Dyet LE, Kennea N, Counsell SJ, Maalouf EF, Ajayi-Obe M, Duggan PJ, Harrison M, Allsop JM, Hajnal J, Herlihy AH, Edwards B, Laroche S, Cowan FM, Rutherford MA, Edwards AD (2006) Natural history of brain lesions in extremely preterm infants studied with serial magnetic resonance imaging from birth and neurodevelopmental assessment. Pediatrics 118:536-548

12. Counsell SJ, Shen Y, Boardman JP, Larkman DJ, Kapellou O, Ward P, Allsop JM, Cowan FM, Hajnal JV, Edwards AD, Rutherford MA (2006) Axial and radial diffusivity in preterm infants who have diffuse white matter changes on magnetic resonance imaging at term-equivalent age. Pediatrics 117:376386

13. Ramenghi LA, Fumagalli M, Righini A, Bassi L, Groppo M, Parazzini C, Bianchini E, Triulzi F, Mosca F (2007) Magnetic resonance imaging assessment of brain maturation in preterm neonates with punctate white matter lesions. Neuroradiology 49:161-167

14. Leijser LM, Liauw L, Veen S, de Boer IP, Walther FJ, van WezelMeijler G (2008) Comparing brain white matter on sequential cranial ultrasound and MRI in very preterm infants. Neuroradiology 50:799-811

15. Volpe JJ (2003) Cerebral white matter injury of the premature infant - more common than you think. Pediatrics 112:176-180

16. Inder TE, Warfield SK, Wang H, Hüppi PS, Volpe JJ (2005) Abnormal cerebral structure is present at term in premature infants. Pediatrics 115:286-294

17. Inder TE, Huppi P, Warfield S, Kikinis R, Zientara GP, Barnes PD, Jolesz F, Volpe JJ (1999) Periventricular white matter injury in the premature infant is followed by a reduction in cerebral cortical gray matter volume at term. Ann Neurol 46:775-760

18. Thompson DK, Warfield SK, Carlin JB, Pavlovic M, Wang HX, Bear M, Kean MJ, Doyle LW, Egan GF, Inder TE (2007) Perinatal 
risk factors altering regional brain structure in the preterm infant. Brain 130:667-677

19. van Wezel-Meijler G (2007) Neonatal cranial ultrasonography. Springer, Berlin

20. Leijser LM, de Bruïne FT, Steggerda SJ, van der Grond J, Walther FJ, van Wezel-Meijler G (2009) Brain imaging findings in very preterm infants throughout the neonatal period: part I. Incidences and evolution of lesions, comparison between ultrasound and MRI. Early Hum Dev 85:101-109

21. van Wezel-Meijler G, van der Knaap M, Sie LTL, Oosting J, van Amerongen AH, Cranendonk A, Lafeber HN (1998) Magnetic resonance imaging of the brain in premature infants during the neonatal period. Normal phenomena and reflection of mild ultrasound abnormalities. Neuropediatrics 29:89-96

22. Boxma A, Lequin M, Ramenghi LA, Kros M, Govaert P (2005) Sonographic detection of the optic radiation. Acta Paediatr 94:1455-1461

23. de Vries LS, Roelants-van Rijn AM, Rademaker KJ, Van Haastert IC, Beek FJ, Groenendaal F (2001) Unilateral parenchymal haemorrhagic infarction in the preterm infant. Eur $\mathrm{J}$ Paediatr Neurol 5:139-149

24. Levene MI (1981) Measurement of the growth of the lateral ventricles in preterm infants with real-time ultrasound. Arch Dis Child 56:900-904

25. van Wezel-Meijler G, Leijser LM, de Bruïne FT, Steggerda SJ, van der Grond J, Walther FJ (2009) Magnetic resonance imaging of the brain in newborn infants: practical aspects. Early Hum Dev 85:85-92

26. Roelants-van Rijn AM, Groenendaal F, Beek FJA, Eken P, van Haastert IC, de Vries LS (2001) Parenchymal brain injury in the preterm infant: comparison of cranial ultrasound, MRI and neurodevelopmental outcome. Neuropediatrics 32:80-89

27. Ment LR, Bada HS, Barnes P, Grant PE, Hirtz D, Papile LA, Pinto-Martin J, Rivkin M, Slovis TL (2002) Practice parameter: neuroimaging of the neonate. Report of the quality standards subcommittee of the American Academy of Neurology and the Practice Committee of the Child Neurology Society. Neurology 58:1726-1738 